\title{
Sinais macroscópicos de deterioração de medicamentos em estoques caseiros
}

\author{
Macroscopic signs of deterioration of medicines in home stocks \\ Signos macroscópicos de deterioro de medicamentos en los almacenamientos domiciliarios
}

Recebido: 25/05/2021 | Revisado: 01/06/2021 | Aceito: 07/06/2021 | Publicado: 22/06/2021

João Pedro Vasconcelos Paolinelli

ORCID: https://orcid.org/0000-0001-7981-6763

Universidade Federal de São João del-Rei, Brasil

E-mail: joaopedro.paolinelli@gmail.com

Diego Bruno Morais

ORCID: https://orcid.org/0000-0002-8310-1813

Universidade Federal de São João del-Rei, Brasil E-mail: moraisdiegobruno@gmail.com

William Neves Oliveira

ORCID: https://orcid.org/0000-0003-3764-4291

Universidade Federal de São João del-Rei, Brasil E-mail: williamnevesufsj@gmail.com

Máyra Rodrigues Fernandes

ORCID: https://orcid.org/0000-0002-4135-3131

Universidade Federal de São João del-Rei, Brasil E-mail: mayra.rfernandes@gmail.com

Rafaela Silva Rocha

ORCID: https://orcid.org/0000-0001-6091-9180

Universidade Federal de Minas Gerais, Brasil E-mail: rafaelarocha.farma@gmail.com

Roberta Carvalho Figueiredo

ORCID: https://orcid.org/0000-0001-6462-3504

Universidade Federal de São João del-Rei, Brasil

E-mail: robertafigueiredo@ufsj.edu.br

André Oliveira Baldoni

ORCID: https://orcid.org/0000-0001-6379-0415

Universidade Federal de São João del-Rei, Brasil

E-mail: andrebaldoni@ufsj.edu.br

Mariana Linhares Pereira

ORCID: https://orcid.org/0000-0002-3214-2789

Universidade Federal de São João del-Rei, Brasil

E-mail: marianapereira@ufsj.edu.br

\begin{abstract}
Resumo
$\mathrm{Na}$ literatura ainda são escassos os estudos que avaliam a qualidade dos medicamentos armazenados nas residências dos pacientes. Assim, este estudo teve como objetivo descrever a presença de sinais macroscópicos de deterioração em formas farmacêuticas encontradas nos estoques caseiros de medicamentos. Trata-se de um estudo observacional descritivo que utilizou dados de uma entrevista realizada entre agosto de 2014 e julho de 2016 nas residências de 423 usuários atendidos na atenção primária à saúde (APS) em um município mineiro de médio porte. A avaliação da deterioração foi realizada por dois pesquisadores previamente treinados que seguiram parâmetros estabelecidos na literatura. A descrição dos resultados foi feita por meio de frequência absoluta e relativa. 21 (5\%) das 423 residências visitadas apresentaram pelo menos um medicamento com sinal macroscópico de deterioração. Encontrou-se uma frequência de 7,5\% $(\mathrm{n}=24)$ de medicamentos deteriorados, sendo esta deterioração presente tanto em medicamentos que se encontravam dentro $3,2 \%(\mathrm{n}=7)$ quanto fora do prazo de validade 4,8\% $(\mathrm{n}=4)$. Dos outros 13 medicamentos que estavam deteriorados, não foi possível constatar a data de validade. $O$ estudo evidenciou uma baixa frequência de deterioração macroscópica nos medicamentos encontrados nos estoques caseiros dos pacientes. Destaca-se que a deterioração não ocorreu apenas em medicamentos vencidos, fato que pode ser explicado pelas más condições de armazenamento nos domicílios.
\end{abstract}

Palavras-chave: Estabilidade de medicamentos; Prazo de validade de produtos; Estoque de medicamentos; Uso de medicamentos; Saúde pública.

\footnotetext{
Abstract

There are few studies in the literature that assess the quality of medicines stored in patients homes. Thus, this study aimed to describe the presence of macroscopic signs of deterioration in pharmaceutical forms found in home stocks medicines. This is a descriptive observational study that used data from an interview conducted between August 2014
} 
and July 2016 in the homes of 423 users assisted in primary health care (PHC) in a medium-sized city in Minas Gerais. The assessment of deterioration was performed by two previously trained researchers who followed parameters established in the literature. The description of the results was made using absolute and relative frequency. $21(5 \%)$ of the 423 households visited had at least one medicine with a macroscopic sign of deterioration. A frequency of $7.5 \%(n=24)$ of deteriorated medicines was found, with this deterioration being present both in medicines that were within $3.2 \%(n=7)$ and out of date $4.8 \%(n=4)$. Of the other 13 medicines that were deteriorated, it was not possible to verify the expiration date. The study evidenced a low frequency of macroscopic deterioration in the medicines found in the patients home stock. It is noteworthy that the deterioration did not only occur in expired medicines, a fact that can be explained by the poor storage conditions at home.

Keywords: Drug stability; Date of validity of products; Drug storage; Drug utilization; Public health.

\section{Resumen}

Existen pocos estudios en la literatura que evalúen la calidad de los medicamentos mantenidos en los hogares de los pacientes. Por ello, este estudio tuvo como objetivo describir la presencia de signos macroscópicos de deterioro en las formas farmacéuticas que se encuentran en el almacenamiento domiciliario de medicamentos. Se trata de un estudio observacional descriptivo que utilizó datos de una entrevista realizada entre agosto de 2014 y julio de 2016 en los hogares de 423 usuarios atendidos en atención primaria de salud (APS) en una ciudad mediana de Minas Gerais. La evaluación del deterioro fue realizada por dos investigadores que fueran previamente capacitados y que siguieron los parámetros establecidos en la literatura. La descripción de los resultados se realizó mediante frecuencia absoluta y relativa. Un 21 (5\%) de los 423 hogares visitados tenían al menos un medicamento con un signo macroscópico de deterioro. Se encontró una frecuencia de 7,5\% $(n=24)$ de medicamentos deteriorados, siendo que este deterioro estuvo presente tanto en los medicamentos que se encontraban dentro del plazo da validad $(3,2 \%, \mathrm{n}=7)$ como los que se encontraban fuera del plazo de validad $(4,8 \%, \mathrm{n}=4)$. De los otros 13 medicamentos que se deterioraron, no fue posible verificar la fecha de vencimiento. El estudio evidenció una baja frecuencia de deterioro macroscópico en los medicamentos encontrados en los almacenamientos domiciliarios de los pacientes. Sin embargo, fue notable que el deterioro no solo ocurrió en los medicamentos caducados, hecho que puede explicarse por las malas condiciones de almacenamiento en el hogar.

Palabras clave: Estabilidad de medicamentos; Fecha de caducidad de productos; Almacenaje de medicamentos; Utilización de medicamentos; Salud pública.

\section{Introdução}

Com as políticas públicas de saúde instituídas no Brasil, desde a promulgação da Constituição Federal em 1988 e da Lei Orgânica da Saúde em 1990, a população tem acesso aos medicamentos e insumos essenciais por meio dos componentes básico, especializado e estratégico da Assistência Farmacêutica, assim como a serviços farmacêuticos, previstos na Política Nacional de Assistência Farmacêutica (PNAF) que é parte integrante da Política Nacional de Saúde (PNS) (1990; 2004a; Brasil, 2016). Ademais, por meio do Programa Farmácia Popular do Brasil (Brasil, 2004b), o governo federal, em parceria com a iniciativa privada, propicia o acesso à população com medicamentos sem custos ou subsidiados em até 90\% (Brasil, 2011). Uma outra forma, não convencional, de acesso a medicamentos pelo Sistema Único de Saúde (SUS) é via demandas judiciais (Santos et al., 2018).

As amplas possibilidades de aquisição, no entanto, não garantem o uso correto do medicamento (Schneider \& Schulte, 2013). Uma forma de avaliar o acesso, as condições de armazenamento, os hábitos de consumo e analisar o seu uso seguro e racional, é verificar a disponibilidade destes medicamentos nos próprios domicílios. Entende-se como Estoque Domiciliar de Medicamentos ou Farmácia Caseira, a guarda de medicamentos decorrentes tanto de sobras de tratamentos anteriores, quanto por medicamentos em uso ou fora de uso, prescritos ou provenientes da automedicação (Dal Pizzol et al., 2006). O Estoque Domiciliar de Medicamentos é uma realidade e pode contribuir para o uso irracional de medicamentos, pois a maioria das pessoas não estão cientes sobre as boas condições de armazenamento, expiração do prazo de validade, sinais macroscópicos de deterioração e seu descarte correto (Bueno, Weber \& Oliveira, 2009).

A deterioração pode ser comum no estoque caseiro de medicamentos, uma vez que o seu mau acondicionamento e o seu vencimento podem comprometer a estabilidade. A redução da estabilidade pode ser ocasionada por fatores extrínsecos, como: temperatura, umidade e luz e/ou por fatores intrínsecos ao produto (Allen, Popovich \& Ansel, 2013). Ambos os fatores 
podem levar a uma decomposição química comprometendo o efeito terapêutico. Podem ocorrer ainda, perda de potência devido a uma diminuição direta do teor do princípio ativo, formação de subprodutos de degradação, podendo ser tóxicos para o organismo, além de deterioração macroscópica na formulação farmacêutica (Allen, Popovich \& Ansel, 2013; Haywood \& Glass, 2016; Brasil, 2012a).

Após a expiração da data de validade, a estabilidade dos medicamentos não é mais garantida pelo fabricante e as chances de os sinais macroscópicos de deterioração ficarem aparentes são maiores. Contudo, ao nosso conhecimento, ainda não existem estudos que avaliem a qualidade dos medicamentos armazenados nas residências dos pacientes. Neste contexto, o presente estudo teve como objetivo descrever a frequência de deterioração macroscópica em cada forma farmacêutica encontrada nos estoques caseiros de medicamentos de indivíduos atendidos na Atenção Primária à Saúde (APS).

\section{Metodologia}

Trata-se de um estudo observacional descritivo, que faz parte de um estudo maior (Fernandes et al., 2020b), que utilizou dados de uma entrevista realizada, entre agosto de 2014 a julho de 2016, nas residências de 423 usuários atendidos na APS de Divinópolis, Minas Gerais, cuja população consiste em 217.404 mil habitantes (Brasil, 2012 b). O município possui 34 unidades APS e cinco farmácias que distribuem medicamentos do componente básico da Assistência Farmacêutica.

Foram sorteadas aleatoriamente 15 unidades da APS. Unidades de APS localizadas em zona rural e com alta taxa de violência urbana, foram excluídas do sorteio, por limitar a viabilização do estudo. Os indivíduos foram convidados a participarem do estudo quando estavam presentes nas unidades de saúde sorteadas. Posteriormente, o entrevistador agendou a visita domiciliar para avaliar o estoque de medicamentos. Foi utilizado um questionário estruturado (Os autores, 2014) previamente testado e validado para coletar as informações dos indivíduos e durante a entrevista foi possível avaliar a presença de deterioração macroscópica em cada forma farmacêutica encontrada nos estoques caseiros. Todos os participantes entrevistados assinaram o Termo de Consentimento Livre e Esclarecido (TCLE) (Fernandes et al., 2020b).

A deterioração macroscópica foi avaliada por dois pesquisadores previamente treinados, no momento da visita domiciliar, utilizando parâmetros estabelecidos na literatura para avaliar a presença de deterioração macroscópica:

a) Comprimidos: presença de rachaduras de superfície; perda de cor; pó em excesso; aderência entre as unidades; depósitos de cristais sobre o produto; lascas; manchas (Marin, Luiza, de Castro \& Santos, 2003; Brasil, 2010);

b) Drágeas: manchas superficiais, rachaduras e fissuras (Marin, Luiza, de Castro \& Santos, 2003; Brasil, 2010);

c) Cápsulas: qualquer sinal de mudança na consistência ou aparência física da cápsula (amolecimento ou endurecimento) (Marin et al., 2003; Brasil, 2010);

d) Cremes e pomadas: formação de grânulos e grumos; textura arenosa; separação de fases; mudança na consistência; diminuição do volume por perda de água; presença de líquido ao pressionar a bisnaga (Marin et al.,2003; Brasil, 2010);

e) Soluções: mudança de cor; precipitação; vazamento; formação de cristais; formação de gases; turbidez (Marin et al., 2003; Brasil, 2010);

f) Suspensões: cheiro forte; mudança de cor; liberação de gases; precipitação; intumescimento; formação de grumos (Marin et al., 2003; Brasil, 2010).

Os parâmetros de avaliação de deterioração de outras formas farmacêuticas não foram descritos por não terem sido encontradas nas amostras analisadas pelo presente estudo. A análise descritiva foi realizada através da apresentação dos dados em frequência absoluta e relativa. O estudo foi aprovado pelo Comitê de Ética em Pesquisa da Universidade Federal de São João Del-Rei (UFSJ), Campus Centro-Oeste Dona Lindu, pelo CAAE: 3091 2314.0.0.5545, parecer 655.930. 


\section{Resultados}

Das 423 residências visitadas, 5\% $(\mathrm{n}=21)$ apresentaram pelo menos um medicamento com sinal macroscópico de deterioração. E nessas residências, avaliou-se 320 medicamentos, sendo que 7,5\% (n=24) estavam com sinais macroscópicos de deterioração. Do total de medicamentos avaliados, 69,4\% (n=222) não estavam vencidos, 26,6\% (n=85) com o prazo de validade expirado e 4\% (n=13) não foi possível constatar a data de validade em consequência das suas condições de armazenamento, ausência de embalagem primária e/ou secundária.

Dentre os medicamentos vencidos $(n=85)$, a frequência de deteriorados foi de $4,8 \%(n=4)$ e entre os não vencidos $(n=222)$ foi de 3,2\% (n=7). Além disso, os 13 medicamentos em que não foi possível constatar a data de validade, também estavam deteriorados (Tabela 1).

Tabela 1. Medicamentos com sinais macroscópicos de deterioração encontrados em estoques caseiros de usuários da Atenção Primária à Saúde de município mineiro, Brasil, entre 2014 e $2016(\mathrm{n}=320)$.

\begin{tabular}{|c|c|c|c|c|c|c|}
\hline \multirow{3}{*}{$\begin{array}{c}\text { Forma } \\
\text { Farmacêutica }\end{array}$} & \multicolumn{2}{|c|}{$\begin{array}{l}\text { Vencido } \\
(n=85)\end{array}$} & \multicolumn{2}{|c|}{$\begin{array}{l}\text { Não vencido } \\
\quad(\mathbf{n}=\mathbf{2 2 2})\end{array}$} & \multicolumn{2}{|c|}{$\begin{array}{l}\text { Validade não identificada } \\
\qquad(n=13)\end{array}$} \\
\hline & $\begin{array}{c}\text { Não } \\
\text { deteriorado }\end{array}$ & Deteriorado & $\begin{array}{c}\text { Não } \\
\text { deteriorado }\end{array}$ & Deteriorado & $\begin{array}{c}\text { Não } \\
\text { deteriorado }\end{array}$ & Deteriorado \\
\hline & \multicolumn{6}{|c|}{ n (\%) } \\
\hline Cápsula & $10(11,7)$ & $2(2,4)$ & $28(12,6)$ & $1(0,5)$ & & \\
\hline Comprimido & $66(77,5)$ & - & $151(68)$ & - & - & $13(100)$ \\
\hline Creme/Pomada & $2(2,4)$ & - & $12(5,4)$ & $2(0,9)$ & - & - \\
\hline Drágea & - & - & $2(0,9)$ & $2(0,9)$ & - & - \\
\hline Solução & $3(3,6)$ & $1(1,2)$ & $16(7,2)$ & - & - & - \\
\hline Suspensão & - & $1(1,2)$ & $6(2,7)$ & $2(0,9)$ & - & - \\
\hline Total & $81(95,2)$ & $4(4,8)$ & $215(96,8)$ & $7(3,2)$ & 0 & $13(100)$ \\
\hline
\end{tabular}

\section{Discussão}

Os resultados indicam que no estoque caseiro das residências há uma baixa frequência de medicamentos que apresentaram sinais macroscópicos de deterioração. Esse achado sugere que a integridade do aspecto macroscópico, presente na maioria dos medicamentos, mesmo após a expiração do prazo de validade, pode ser um dos motivos que justificam as pessoas a estocarem estes medicamentos em casa. Além disso, este resultado aponta para a necessidade de questionamentos sobre o real prazo de validade dos medicamentos e da funcionalidade e aplicabilidade da análise macroscópica como forma de identificar o sinal de que o medicamento está vencido.

Nesse contexto, é importante ressaltar que nos Estados Unidos existe o Programa de Extensão do Prazo de Validade "Shelf-life Extension Program" (SLEP), gerenciado pela Food and Drug Administration (FDA) em parceria com o Department of Defense (DoD), que avalia a estabilidade em longo prazo dos estoques de medicamentos federais. Seus dados sustentam a afirmação de que o uso dos medicamentos pode ser estendido além da data de validade original (Khan et al., 2014). Ademais, Zilker et al. (2019), levantaram evidências por meio de uma revisão sistemática, de que muitos medicamentos possuem uma estabilidade muito além do prazo de validade rotulado pelo fabricante. No entanto, essa estabilidade adicional encontrada em ambos os estudos citados é altamente variável de produto a produto e lote a lote, e os dados do programa americano só podem ser generalizados a produtos cuidadosamente armazenados e que estejam em suas embalagens originais (Lyon et al., 2006) o que normalmente não acontece no armazenamento domiciliar (Martins et al., 2017; Fernandes et al., 2020a; Fernandes et al., 2020b). Diante disso, não podemos considerar que os medicamentos que fazem parte do estoque caseiro possuem essa 
estabilidade adicional evidenciada nestes estudos, uma vez que o armazenamento nos domicílios, muitas vezes, não acontece de maneira correta.

Instigados pelos resultados do SLEP americano, um ensaio pré-clínico em camundongos foi realizado com o objetivo de avaliar a efetividade e segurança de três anti-inflamatórios não-esteroidais (AINEs) - Ibuprofeno, Piroxicam e Diclofenaco de Potássio - vencidos. Ao contrário do Piroxicam que demonstrou atividade anti-inflamatória mais alta que a não expirada, os outros dois AINEs exibiram um nível mais baixo de atividade do que seus equivalentes não expirados. A segurança foi avaliada analisando os níveis de enzimas hepáticas Alanina-Amino-Transferase (ALT) e Aspartato-Amino-Transferase (AST), ureia e bilirrubina, não havendo alterações significativamente importantes entre os grupos comparados (Ihekwereme, Chidebelu, Nwadiliorah, 2017). Ressalta-se que essa diferença na atividade dos medicamentos estudados pode ser resultante de interações medicamentosas com excipientes ou condições inadequadas de armazenamento, levando a alteração química e formação de subprodutos de degradação (Chaudhari \& Patil, 2012).

É importante destacar, também, que o presente estudo evidenciou que a deterioração não ocorre apenas com medicamentos vencidos. Este fato pode ser explicado pelas más condições de acondicionamento destes medicamentos nos domicílios. Muitos estudos descrevem o estoque caseiro de medicamentos em cenários diferentes e, em todos aqueles que tivemos acesso, a maioria dos medicamentos se encontrava em condições inapropriadas de armazenamento, como na cozinha, e banheiro, expostos à luz e umidade (Schneider \& Schulte, 2013; Fernandes et al., 2020a; Milanez et al., 2013; Schwingel, 2015;). Sabe-se ainda que a deterioração macroscópica é um importante indicativo para se identificar a perda da qualidade do medicamento, no entanto, alguns estudos citados no trabalho de Albert et al. (2017) sugerem que nem sempre uma degradação química resulta em degradação macroscópica do produto. Neste contexto, recomenda-se que o profissional da saúde oriente os pacientes quanto ao armazenamento correto dos medicamentos nos domicílios, para se evitar a perda da qualidade antes do vencimento e os aconselhe a não utilizarem os vencidos, uma vez que, a ausência de sinais macroscópicos de deterioração, não assegura que o produto está em boas condições de uso.

Aponta-se como limitação do estudo, que os resultados não podem ser generalizados para usuários do sistema de saúde privado e para moradores de zonas rurais (Fernandes et al., 2020b). No entanto, ao melhor do nosso conhecimento, este foi o primeiro estudo que avaliou a presença de deterioração nos estoques caseiros de medicamentos de pacientes e trouxe importantes reflexões baseadas em evidências científicas que auxiliarão os profissionais da saúde na orientação de seus pacientes sobre o uso seguro e racional de medicamentos.

\section{Conclusão}

Os resultados encontrados evidenciaram uma baixa frequência, porém importante, de deterioração macroscópica de medicamentos dos Estoques Caseiros dos pacientes. Além disso, o presente estudo sugere que a deterioração não ocorre apenas em medicamentos vencidos, fato que pode ser explicado pelas más condições de armazenamento nos domicílios. Estes achados apontam para a necessidade de realização de novas investigações no sentido de gerar evidências sobre os reais prazos de validade dos medicamentos e da funcionalidade da análise macroscópica dos medicamentos. Isso deve ser tema de discussão e investigação junto a todos os atores envolvidos na logística de medicamentos, sendo as agências reguladoras, indústria farmacêutica, serviços de saúde, comunidade, gestores e centros de pesquisas. 


\section{Agradecimentos}

Agradecemos a Universidade Federal de São João del-Rei (UFSJ) e ao Conselho Nacional de Desenvolvimento Científico e Tecnológico (CNPq) pelas bolsas concedidas. O presente trabalho foi realizado com apoio da Coordenação de Aperfeiçoamento de Pessoal de Nível Superior - Brasil (CAPES) - Código de Financiamento 001.

\section{Referências}

Albert, V., Lanz, M., Imanidis, G., Hersberger, K.E. \& Arnet, I. (2017). Stability of medicines after repackaging into multicompartment compliance aids: eight criteria for detection of visual alteration. Drugs Ther Perspect. 2017;33(10):487-96. DOI: 10.1007/s40267-017-0431-9.

Allen, L.V., Popovich, N.G. \& Ansel, H.C. (2013). Formas Farmacêuticas e Sistemas de Liberação de Fármacos. 9 ed. Porto Alegre: Artmed. ISBN: 9788565852852 .

Brasil. (1990). Presidência da República. Casa Civil. Subchefia para Assuntos Jurídicos. Lei no 8.080, de 19 de setembro de 1990 . Dispõe sobre as condições para a promoção, proteção e recuperação da saúde, a organização e o funcionamento dos serviços correspondentes e dá outras providências. Brasília (DF): 19 de setembro de 1990 [citado 2020 Dez 17]. http://www.planalto.gov.br/ccivil_03/leis/18080.htm.

Brasil. (2004a). Ministério da Saude. Conselho Nacional de Saúde. Resolução n³38, de 06 de Maio de 2004. Brasília (DF). bvsms.saude.gov.br/bvs/saudelegis/cns/2004/res0338_06_05_2004.html.

Brasil. (2004b). Presidência da República. Casa Civil. Subchefia para Assuntos Jurídicos. Decreto n 5090, 20 de Maio de 2004. Regulamenta a Lei no 10.858, de 13 de abril de 2004, e institui o programa "Farmácia Popular do Brasil", e dá outras providências. Brasília (DF). http://www.planalto.gov.br/ccivil_03/_Ato2004-2006/2004/Decreto/D5090.htm.

Brasil. (2010). Ministério da Saúde. Secretaria de Vigilância em Saúde. Departamento de DST, Aids e Hepatites Virais. Protocolo de Assistência Farmacêutica em DST/HIV/Aids. Recomendações do Grupo de Trabalho de Assistência Farmacêutica. Brasília (DF). file:///C:/Users/Jo\%C3\%A3o\%20Pedro/Downloads/protocolo_internet.pdf .

Brasil. (2011). Ministério da Saude. Conselho Nacional de Saúde. Portaria nº184, de fevereiro de 2011. Dispões sobre o Programa Farmácia Popular do Brasil. Brasília (DF). http://bvsms.saude.gov.br/bvs/saudelegis/gm/2011/prt0184_03_02_2011.htm.

Brasil. (2012). Ministério da Saúde. DATASUS. Departamento de Informática do SUS. Sistema de informática do Sistema Único de Saúde.População residente em Divinópolis. Brasília (DF). http://tabnet.datasus.gov.br/cgi/deftohtm.exe?ibge/cnv/popbr.def.

Brasil. (2016). Senado Federal. Secretaria de Editoração e Publicações. Coordenação de Edições Técnicas. Constituição da República Federativa do Brasil: texto constitucional promulgado em 5 de outubro de 1998, com alterações determinadas pelas Emendas Constirucionais de revisão ${ }^{\circ}{ }^{1}$ a 6/94, pelas Emendas Constitucionais $\mathrm{n}^{\circ} 1 / 92$ a 91/2016 e pelo Decreto Legislativo $\mathrm{n}^{\circ}$ 186/2008. Brasília (DF): Senado Federal, Coordenação de Edições Técnicas. https://www2.senado.leg.br/bdsf/bitstream/handle/id/518231/CF88_Livro_EC91_2016.pdf.

Brasil. (2019). Ministério da Saúde. Agência Nacional de Vigilância Sanitária. Diretoria Colegiada Resolução nº 318 , de 6 de novembro de 2019. Brasília (DF). https://www.in.gov.br/en/web/dou/-/resolucao-rdc-n-318-de-6-de-novembro-de-2019-226513805.

Bueno, C.S., Weber, D. \& Oliveira, K.R. (2009). Farmácia caseira e descarte de medicamentos no bairro Luiz Fogliatto do município de Ijuí - RS. Rev Cienc Farm Basica Apl. 30(2):203-10. https://rcfba.fcfar.unesp.br/index.php/ojs/article/view/447.

Chaudhari, S.P. \& Patil, P.S. (2012). Pharmaceutical excipients: a review. Int J Adv Pharmacy Biol Chem. 1(1):21-34. http://www.ijapbc.com/files/4.pdf.

Dal Pizzol, T.S., Piccoli, A., Brugnera, Q., Schenkel, E.P. \& Mengue, S.S. (2006). Análise dos estoques domiciliares de medicamentos essenciais no Sul do Brasil. Acta Farm. Bonaerense. 25(04):601-7. http://sedici.unlp.edu.ar/handle/10915/6887.

Fernandes, M.R., Figueiredo, R.C., Silva, L.G.R., Rocha, R.S. \& Baldoni, A.O. (2020a). Armazenamento e descarte dos medicamentos vencidos em farmácias caseiras: problemas emergentes para a saúde pública. Eistein (São Paulo). 18:1-6. http://dx.doi.org/10.31744/ einstein_journal/2020AO5066.

Fernandes, M.R., Rocha, R.S., Silva, I.R., Figueiredo, R.C \& Baldoni, A.O. (2020b). Prevalência e fatores associados à presença de medicamentos vencidos em estoques caseiros. Cad Saúde Coletiva. 28(3):101-10. https://doi.org/10.1590/1414-462X202028030535.

Haywood, A.D. \& Glass, B.D. (2016). Evidence of stability of medicines repackaged in compliance aids: a review. Curr Drug Saf. 11(1):69-77. DOI:10.2174/1574886310666150928104931.

Ihekwereme, C.P., Chidebelu, C.C. \& Nwadiliorah, L.C. (2017). Evaluation of the effectiveness of expired anti-inflammatory medicines in tropical africa using mice. Am J Drug Deliv Ther. 4(1):3. https://www.imedpub.com/articles/evaluation-of-the-effectiveness-of-expired-antiinflammatory-medicines-intropical-africa-using-mice.php?aid=20443.

Khan, S.R., Kona, R., Faustino, P.J., Gupta, A., Taylor, J.S., Porter, D.A. \& Poter, M. K. (2014). United States Food and Drug Administration and Department of Defense shelf-life extension program of pharmaceutical products: progress and promise. J Pharm Sci. 2014;103(5):1331-6. DOI: 10.1002/jps.23925.

Lyon, R.C., Taylor, J.S., Porter, D.A., Prasanna, H.R. \& Hussain, A.S. (2006). Stability profiles of drug products extended beyond labeled expiration dates. J Pharm Sci. 2006;95(7):1549-60. DOI: 10.1002/jps.20636.

Marin, N., Luiza, V.L., Castro, C.G. \& Santos, S.M. (2003). Assistência farmacêutica para gerentes municipais. Rio de Janeiro:OPAS/OMS; p. 373. https://www.cff.org.br/userfiles/84\%20-\%20MARIN\%20N\%20ET\%20AL\%20Assistencia\%20Farmaceutica\%20para\%20gerentes\%20municipais_2003.pdf. 
Research, Society and Development, v. 10, n. 7, e28910716517, 2021

(CC BY 4.0) | ISSN 2525-3409 | DOI: http://dx.doi.org/10.33448/rsd-v10i7.16517

Martins, R.R., Farias, A.D., Oliveira, Y.M., Diniz, R.D. \& Oliveira, A.G. (2017). Prevalence and risk factors of inadequate medicine home storage: a community-based study. Rev Saude Publica. 2017;51:95. https://doi.org/10.11606/S1518-8787.2017051000053.

Milanez, M.C., Stutz, E., Rosales, T.O., Penteado, A.J., Perez, E., Cruciol, J.M. Pereira, E. M. \& Bovo, F. (2013). Avaliação dos Estoques Domiciliares de Medicamentos em uma Cidade do Centro-Sul do Paraná. Rev Ciênc Méd Biol. 2013;12(3):283. https://repositorio.ufba.br/ri/bitstream/ri/23088/1/3_v.12_3.pdf.

Santos, E.C., Teixeira, C.R., Zanetti, M.L., Istilli, P.T., Pereira, L.H.T.R \& Torquato, M.T. (2018). Health judicialization: access to treatment for users with diabetes mellitus. Texto Contexto Enferm. 27(1):1-7. https://doi.org/10.1590/0104-070720180000800016.

Schneider AP, Schulte I. (2013). Estoque domiciliar de medicamentos na zona urbana do município de Venâncio Aires - RS. Cinergis.14(4):176-80. DOI: http://dx.doi.org/10.17058/cinergis.v14i4.4065.

Schwingel, D., de Souza, J., Simonetti, E., Rigo, M.P., Ely, L.S., de Castro, L.C. Fernandes, L. C. \& Kauffmann, C. (2015). Farmácia caseira x uso racional de medicamentos. Rev Cad Pedag. 12(3):117-30. http://www.univates.br/revistas/index.php/cadped/article/view/973/961.

Zilker M, Sörgel F, Holzgrabe U. (2019). A systematic review of the stability of finished pharmaceutical products and drug substances beyond their labeled expiry dates. J Pharm Biomed Anal. 2019;166:222-35. DOI:10.1016/j.jpba.2019.01.016. 